\title{
Probabilistic Approximation via Spatial Derivation of Some Nonlinear Parabolic Evolution Equations
}

\author{
B. Jourdain
}

ENPC-CERMICS, 6-8 av Blaise Pascal, Cité Descartes, Champs sur Marne, 77455 Marne-la-Vallée Cedex 2, France - jourdain@cermics.enpc.fr

Summary. For some parabolic equations with a local nonlinearity, a suitable spatial derivation leads to a Fokker-Planck equation with a nonlocal nonlinearity. In this paper we present a review of the particle methods obtained by replacing the nonlinearity in this Fokker-Planck equation by interaction. We are interested in the convergence results for the particle approximations of the original equations and give the milestones of their proofs.

\section{Introduction}

Let us justify the key idea leading to the probabilistic interpretation of the parabolic evolution equations treated in this paper on the simple example of the viscous Burgers equation

$$
\partial_{t} u(t, x)=\nu \partial_{x x} u(t, x)-\partial_{x}(u(t, x))^{2},(t, x) \in R_{+} \times R .
$$

Following [10] [31] [26] [27], this equation can be seen as the Fokker-Planck equation associated with a diffusion process with diffusion coefficient equal to $\nu$ and with drift coefficient given by the solution $u$ itself. This leads to the following stochastic differential equation nonlinear in the sense of McKean since the drift coefficient at time $t$ is the density of the solution at the same time :

$$
d X_{t}=\sqrt{2 \nu} d W_{t}+u\left(t, X_{t}\right) d t \text { where } \forall t \geq 0, u(t, x) \text { is the density of } X_{t} .
$$

Here $\left(W_{t}\right)_{t \geq 0}$ is a standard Brownian motion.

Of course, because of the nonlinearity $u$ in the drift term, it is not possible to simulate this stochastic differential equation. But one can simulate systems of $n$ particles where the nonlinearity is replaced by interaction. More precisely, the particles are driven by independent Brownian motions. And the drift coefficient of the $i$-th particle $(1 \leq i \leq n)$ is obtained as the empirical density of 
the system estimated at the position of the $i$-th particle itself. From a numerical point of view, this leads to a difficulty, since the numerical approximation of a density function is unstable.

The approach proposed by Bossy and Talay [7] [8] for the viscous Burgers equation enables to avoid this difficulty. It consists in giving a probabilistic interpretation to the equation satisfied by $v=\partial_{x} u$ :

$$
\partial_{t} u=\nu \partial_{x x} u-2 \partial_{x}(u v) .
$$

Since $v$ is the derivative of $u$, the latter function is the integral of the former. This leads to the following nonlinear stochastic differential equation :

$$
d X_{t}=\sqrt{2 \nu} d W_{t}+2 u\left(t, X_{t}\right) d t \text { where } \forall(t, x) \in R_{+} \times R, u(t, x)=P\left(X_{t} \leq x\right) .
$$

In the associated system with $n$ particles, the drift coefficient of the $i$-th particle is obtained as twice the empirical cumulative distribution fonction of the system computed at the position of this particle :

$$
d X_{t}^{i, n}=\sqrt{2 \nu} d W_{t}^{i}+\frac{2}{n} \sum_{j=1}^{n} 1_{\left\{X_{t}^{j, n} \leq X_{t}^{i, n}\right\}} d t, 1 \leq i \leq n
$$

where $\left(W^{1}, \ldots, W^{n}\right)$ is a $n$-dimensional Brownian motion. Notice that ordering the particles (with computational cost $\mathcal{O}(n \log n)$ ) is enough to compute the drift coefficients of all particles. The solution $u(t, x)$ of the Burgers equation is then approximated by $\frac{1}{n} \sum_{i=1}^{n} 1_{\left\{X_{t}^{i, n} \leq x\right\}}$. This provides a numerical method which may be more efficient than classical ones, when e.g. the viscosity coefficient $\nu$ is small (see [5]).

In the first section of the paper, we show how this approach can be adapted for general one-dimensional parabolic equations. In the second section, we consider spatial domains equal to $R^{d}, d \geq 2$. The last section adresses bounded spatial domains.

\section{One Dimensional Equations}

We are interested in the initial value problem for the following parabolic evolution equation with local nonlinearity

$$
\begin{gathered}
\partial_{t} u(t, x)=\partial_{x x}[\alpha(u(t, x))]-\partial_{x}[\beta(u(t, x))],(t, x) \in R_{+} \times R \\
u(0, x)=u_{0}(x), x \in R
\end{gathered}
$$

where $\alpha, \beta: R \rightarrow R$ are $C^{1}$ functions with $\alpha$ non-decreasing (at least on the interval $\left.\left(\inf _{R} u_{0}(x), \sup _{R} u_{0}(x)\right)\right)$ and $u_{0}: R \rightarrow R$ is a non-constant function with bounded variation i.e. $u_{0}()=.c+\int_{-\infty}^{\cdot} m(d y)$ with $m$ a bounded signed measure on the real line. By modifying the functions $\alpha$ and $\beta$ without changing their smoothness, we may assume without restriction that $c$ is equal to zero and that $m$ is a probability measure when the function $u_{0}$ is monotonic. The following equations of physical interest may be written in the form (1) : 
- the viscous scalar conservation law $\partial_{t} u=\nu \partial_{x x} u-\partial_{x} \beta(u)$ where $\nu>0$ obtained in the case $\alpha(u)=\nu u$.

- the inviscid scalar conservation law $\partial_{t} u+\partial_{x} \beta(u)=0$ obtained in the case $\alpha \equiv 0$.

- the porous medium equation $\partial_{t} u=\partial_{x x} u^{q}$ with $q>1$ obtained in the case $\alpha(u)=u^{q}$ and $\beta \equiv 0$. Then the initial condition $u_{0}$ has to be non-negative.

Before dealing with general initial conditions, we consider monotonic functions $u_{0}$.

\subsection{Monotonic Initial Conditions}

We assume that $u_{0}(x)=m((-\infty, x])$ with $m$ a probability measure on $R$. By spatial derivation of (1), one obtains that $v=\partial_{x} u$ formally solves

$$
\partial_{t} v=\partial_{x x}\left[\alpha^{\prime}(u) v\right]-\partial_{x}\left[\beta^{\prime}(u) v\right], v(0, .)=m .
$$

To obtain a closed equation, one has to express $u$ which appears in $\alpha^{\prime}$ and $\beta^{\prime}$ in terms of $v$. Since $v$ is the spatial derivative of $u\left(\right.$ and $\left.\lim _{x \rightarrow-\infty} u_{0}(x)=0\right)$, one expects that $u(t, x)=\int_{-\infty}^{x} v(t, y)=H * v(t,).(x)$ where $H(x)=1_{\{x \geq 0\}}$ denotes the Heaviside function. Hence $v$ formally solves the following parabolic equation with nonlocal nonlinearity

$$
\partial_{t} v=\partial_{x x}\left[\alpha^{\prime}(H * v) v\right]-\partial_{x}\left[\beta^{\prime}(H * v) v\right], v(0, .)=m .
$$

We are now going to distinguish the non-degenerate case obtained when $\alpha^{\prime}$ is a positive function on the real line (assumption satisfied for the viscous scalar conservation law) and the case of inviscid scalar conservation laws $(\alpha \equiv 0)$.

\section{Non-Degenerate Case}

We assume that $\forall u \in R, \alpha^{\prime}(u)>0$. Let $\left(X_{t}\right)_{t \geq 0}$ and $\mathcal{P}(C([0,+\infty), R))$ denote respectively the canonical process and the space of probability measures on $C([0,+\infty), R)$. Interpreting (2) as a nonlinear Fokker-Planck equation, we associate the following nonlinear martingale problem with it

Definition 1. A probability measure $P \in \mathcal{P}(C([0,+\infty), R))$ with time-marginals $\left(P_{t}\right)_{t \geq 0}$ solves problem $(\mathbf{M P})$ if

$$
\begin{aligned}
& \text { 1. } P_{0}=m \\
& \text { 2. } M_{t}^{\varphi}=\varphi\left(X_{t}\right)-\varphi\left(X_{0}\right)-\int_{0}^{t} \alpha^{\prime}\left(H * P_{s}\left(X_{s}\right)\right) \varphi^{\prime \prime}\left(X_{s}\right)+\beta^{\prime}\left(H * P_{s}\left(X_{s}\right)\right) \varphi^{\prime}\left(X_{s}\right) d s \\
& \text { is a P-martingale for any function } \varphi \in C_{b}^{2}(R) \text {. }
\end{aligned}
$$

If $P$ solves (MP), then the function $t \rightarrow E^{P}\left(M_{t}^{\varphi}\right)$ is constant. One deduces that $t \rightarrow P_{t}$ is a weak solution of (2). Since $\alpha^{\prime}$ is bounded from below by a positive constant on the interval $[0,1]$, according to Theorem 2 [21], $d t$ a.e. $P_{t}$ does not weight points. This ensures that $\alpha^{\prime}\left(H * P_{t}\right) P_{t}\left(\right.$ resp. $\left.\beta^{\prime}\left(H * P_{t}\right) P_{t}\right)$ is 
the spatial derivative of $\alpha\left(H * P_{t}\right)$ (resp. $\left.\beta\left(H * P_{t}\right)\right)$ in the distribution sense on $R_{+}^{*} \times R$. Therefore if $P$ solves (MP), then $H * P_{t}(x)$ is a weak solution of (1).

Consequently when uniqueness holds for weak solutions of (1), uniqueness for problem (MP) is equivalent to uniqueness for a standard martingale problem which follows from exercise 7.3.3 [30]. Uniqueness for weak solutions of the viscous scalar conservation law $(\alpha(u)=\nu u)$ can be checked easily [15]. When $\beta \equiv 0$, the uniqueness result of [9] ensures that (1) has no more than one weak solution $u$ such that $\left\|u(t, .)-u_{0}\right\|_{L^{1}(R)}$ is locally integrable with respect to $t \in R_{+}$and tends to 0 as $t$ tends to 0 . Lemma 1 stated in the Appendix in the framework of Section 1.2 which generalizes the present one, allows to deduce that if $P$ and $Q$ both solve problem (MP), then $\forall(t, x) \in R_{+} \times R$, $H * P_{t}(x)=H * Q_{t}(x)$. Apart from both these cases, to our knowledge, no general result ensures uniqueness of the weak solution of (1).

Because of the presence of the marginal $P_{s}$ in the coefficients at time $s$, it is not possible to simulate the diffusion dynamics given by (MP). But one obtains a simulable system of $n$ particles by replacing $P_{s}$ by the empirical measure of the system at time $s$ in the coefficients :

$$
X_{t}^{i, n}=X_{0}^{i}+\int_{0}^{t} \sqrt{2 \alpha^{\prime}}\left(H * \mu_{s}^{n}\left(X_{s}^{i, n}\right)\right) d W_{s}^{i}+\int_{0}^{t} \beta^{\prime}\left(H * \mu_{s}^{n}\left(X_{s}^{i, n}\right)\right) d s, i \leq n
$$

where $\mu^{n}=\frac{1}{n} \sum_{j=1}^{n} \delta_{X^{j, n}}$ and the initial variables $X_{0}^{1}, \ldots, X_{0}^{n}$ are i.i.d. according to $m$ and independent from the $n$-dimensional Brownian motion $\left(W^{1}, \ldots, W^{n}\right)$. Existence of a weak solution is ensured by [30] exercise 7.3.2. Weak uniqueness follows from [2] since the diffusion matrix is constant on the polyhedrons $\left\{x=\left(x_{1}, \ldots, x_{n}\right) \in R^{n}, x_{\sigma(1)}<x_{\sigma(2)}<\ldots<x_{\sigma(n)}\right\}$ indexed by the permutations $\sigma$ of $\{1, \ldots, n\}$.

Let $\pi^{n}$ denote the law of the empirical measure $\mu^{n}$ considered as a random variable with values in $\mathcal{P}(C([0,+\infty), R))$. Since in view of weak uniqueness, the processes $X^{i, n}, 1 \leq i \leq n$ are exchangeable, the tightness of the sequence $\left(\pi^{n}\right)_{n}$ is equivalent to the tightness of the laws of $X^{1, n}, n \geq 1$ (see [32]) which follows from the boundedness of $\alpha^{\prime}$ and $\beta^{\prime}$ on the interval $[0,1]$. It is not difficult [16] to prove that the weak limit of any converging subsequence gives full weight to the set of probability measures $Q \in \mathcal{P}(C([0,+\infty), R))$ such that $H * Q_{t}(x)$ is a weak solution of (1). In case this equation admits a unique weak solution $u(t, x)$, one deduces [18] :

$$
\forall T>0, \lim _{n \rightarrow+\infty} E \sup _{t \in[0, T]} \int_{R} \frac{\left|u(t, x)-H * \mu_{t}^{n}(x)\right|}{1+x^{2}} d x=0 .
$$

Hence the empirical cumulative distribution functions $H * \mu_{t}^{n}(x)$ of the particle systems converge to $u(t, x)$ as $n$ tend to infinity.

The next step consists in proving that the limit $\pi^{\infty}$ of any converging subsequence of $\left(\pi^{n}\right)_{n}$ gives full weight to solutions of problem (MP). In case uniqueness holds for this problem, one deduces that when $n$ tends to $+\infty$, 
the empirical measures $\mu_{n}$ converge in probability to the constant $P$ where $P$ denotes the unique solution of problem (MP). The choice of the initial distribution of particles ensures that $\pi^{\infty}$ a.s. condition 1 in definition 1 is satisfied. According to [16], the mappings on $\mathcal{P}(C([0,+\infty), R))$ naturally introduced in order to check condition 2 are continuous at any probability measure $Q$ such that $d t$ a.e. $Q_{t}$ does not weight points. Therefore it is enough to check that $\pi^{\infty}$ gives full weight to such probability measures to conclude. For the viscous scalar conservation law, one may deduce this property from estimates of the density of $\left(X_{t}^{1, n}, X_{t}^{2, n}\right)$ obtained by Girsanov theorem [14] [15]. More generally, it is a consequence of the estimate

$$
E\left(\int_{0}^{t}\left|f\left(X_{s}^{1, n}, X_{s}^{2, n}\right)\right| d s\right) \leq C\|f\|_{L^{3}\left(R^{2}\right)} \text { with } C \text { not depending on } n,
$$

which follows from Theorem 2 [21] since $\alpha^{\prime}$ is bounded from below by a positive constant on the interval $[0,1]$ and the coefficients of the stochastic differential equation (3) are globally bounded.

One may also be interested in the asymptotic behaviour of the reordered system $\left(Y^{1, n}, \ldots, Y^{n, n}\right)$ defined in the following way : for any $t \geq 0$, $Y_{t}^{1, n} \leq Y_{t}^{2, n} \leq \ldots \leq Y_{t}^{n, n}$ is the increasing reordering of $\left(X_{t}^{1, n}, \ldots, X_{t}^{n, n}\right)$. According to [16], $\left(Y^{1, n}, \ldots, Y^{n, n}\right)$ is a diffusion process with constant diffusion matrix $\operatorname{diag}\left(\alpha^{\prime}(1 / n), \alpha^{\prime}(2 / n), \ldots, \alpha^{\prime}(n / n)\right)$ and with drift coefficient $\left(\beta^{\prime}(1 / n), \beta^{\prime}(2 / n), \ldots, \beta^{\prime}(n / n)\right)$ normaly reflected at the boundary of the convex subset $\left\{\left(y_{1}, \ldots, y_{n}\right): y_{1} \leq y_{2} \leq \ldots \leq y_{n}\right\}$ of $R^{n}$.

The laws $\bar{\pi}^{n}$ of the empirical measures $\bar{\mu}^{n}=\frac{1}{n} \sum_{i=1}^{n} \delta_{Y^{i, n}}$ are easily proved to be tight [16]. In general $\bar{\mu}^{n}$ is different from $\mu^{n}$, but of course for any non-negative $t$, one has $\bar{\mu}_{t}^{n}=\mu_{t}^{n}$. Hence the limit $\bar{\pi}^{\infty}$ of any converging subsequence of $\left(\bar{\pi}^{n}\right)_{n}$ also gives full weight to the set of probability measures $Q \in \mathcal{P}(C([0,+\infty), R))$ such that $H * Q_{t}(x)$ is a weak solution of (1). The order between particles enables to check that moreover, $\bar{\pi}^{\infty}$ gives full weight to probability measures $Q$ with finite dimensional marginals $Q_{t_{1}, \ldots, t_{k}}$ equal to the image of the Lebesgue measure on the interval $[0,1]$ by $y \rightarrow\left(\left(H * Q_{t_{1}}\right)^{-1}(y), \ldots,\left(H * Q_{t_{k}}\right)^{-1}(y)\right)$ where for any non-negative $t,\left(H * Q_{t}\right)^{-1}(y)=\inf \left\{x: H * Q_{t}(x) \geq y\right\}$ (see [18]). When uniqueness of weak solutions holds for (1), there is a unique probability measure $\bar{P} \in \mathcal{P}(C([0,+\infty), R))$ with both these properties and the sequence $\left(\bar{\mu}^{n}\right)_{n}$ converges in probability to $\bar{P}$. If in addition, the unique weak solution of (1) is continuous on $] 0,+\infty[\times R$, then according to [16], the second property characterizing $\bar{P}$ is equivalent to the fact that $\bar{P}$ gives full weight to sample-paths $X$ such that $t \rightarrow u\left(t, X_{t}\right)$ is constant on $(0,+\infty)$.

The following Theorem summarizes the results obtained above for the viscous scalar conservation law and the equation with no first order term:

Theorem 1. Assume that $\alpha(u)=\nu u$ with $\nu>0$ (resp. that $\alpha^{\prime}$ is a positive function and that $\beta \equiv 0)$. Then (1) admits a unique solution $u$ and this solution is continuous on $(0,+\infty) \times R$ (resp. (1) admits a unique solution 
$u$ such that $\left\|u(t, .)-u_{0}\right\|_{L^{1}(R)}$ is locally integrable with respect to $t \in R_{+}$ and tends to 0 as $t$ tends to 0$)$. As $n$ tend to infinity, the empirical cumulative distribution functions $H * \mu_{t}^{n}(x)$ of the particle systems converge to $u(t, x)$ in the sense given by (4). The empirical measures of the original particles converge in probability to the unique solution $P$ of problem (MP). The empirical measures of the reordered particles converge in probability to the unique $\bar{P} \in \mathcal{P}(C([0,+\infty), R))$ such that $H * \bar{P}_{t}(x)=u(t, x)$ and that $\bar{P}$ a.s. $t \rightarrow u\left(t, X_{t}\right)$ is constant on $(0,+\infty)$ (resp. and that the finite dimensional marginals $\bar{P}_{t_{1}, \ldots, t_{k}}$ are equal to the image of the Lebesgue measure on the interval $[0,1]$ by $\left.y \rightarrow\left(\left(H * \bar{P}_{t_{1}}\right)^{-1}(y), \ldots,\left(H * \bar{P}_{t_{k}}\right)^{-1}(y)\right)\right)$.

Remark 1. (see [16]) In case of the porous medium equation, $\alpha(u)=u^{q}$ with $q>1$ and $\alpha^{\prime}$ vanishes for $u=0$. By combining weak uniqueness for this equation and existence of a solution $u$ with sufficient spatial regularity, one obtains that problem (MP) has no more than one solution $P$ such that $d t$ a.e., $P_{t}$ does not weight points. As $n$ tends to infinity, the empirical cumulative distribution functions $H * \mu_{t}^{n}(x)$ of the particle systems converge to $u(t, x)$ in the sense given by (4). Since $u$ is continuous on $(0,+\infty) \times R$, one deduces that any limit point of the sequence $\left(\pi^{n}\right)_{n}$ gives full weight to probability measures $Q$ such that $d t$ a.e., $Q_{t}$ does not weight points, and then concludes that the empirical measures $\mu^{n}$ converge in probability to the unique solution $P$ of (MP) such that $d t$ a.e., $P_{t}$ does not weight points. Last, the empirical measures $\bar{\mu}^{n}$ of the reordered particles converge in probability to the unique $\bar{P} \in \mathcal{P}(C([0,+\infty), R))$ such that $H * \bar{P}_{t}(x)=u(t, x)$ and that $\bar{P}$ a.s. $t \rightarrow$ $u\left(t, X_{t}\right)$ is constant on $(0,+\infty)$.

\section{The Inviscid Scalar Conservation Law}

We suppose that $\alpha \equiv 0$. Then uniqueness fails to hold for weak solutions of (1). However, according to Kruzkhov's theorem, there is a unique entropic solution characterized by the following inequalities

$$
\partial_{t}|u(t, x)-c|+\partial_{x}(\operatorname{sign}(u(t, x)-c)(\beta(u(t, x))-\beta(c))) \leq 0
$$

holding in the distribution sense on $R_{+} \times R$ for each real constant $c \in[0,1]$. Here $\operatorname{sign}(y)=1_{\{y>0\}}-1_{\{y \leq 0\}}$. The entropic solution is the limit of the solution of the viscous scalar conservation law as the viscosity coefficient $\nu$ vanishes. This suggests to approximate $u(t, x)$ by $H * \mu_{t}^{n}(x)$ where $\mu^{n}$ is the empirical measure of the system

$$
X_{t}^{i, n}=X_{0}^{i}+\sqrt{2 \nu_{n}} W_{t}^{i}+\int_{0}^{t} \beta^{\prime}\left(H * \mu_{s}^{n}\left(X_{s}^{i, n}\right)\right) d s, 1 \leq i \leq n
$$

with small viscosity coefficient $\nu_{n}>0$. Numerical experiments by Bossy and Talay [8] and Bossy, Piperno and Fezoui [5] show the good behaviour of this 
approximation.

The reordered particle system $\left(Y^{1, n}, \ldots, Y^{n, n}\right)$ turns out to be a useful tool for the theoretical study of the limit behaviour of $H * \mu_{t}^{n}(x)=\frac{1}{n} \sum_{i=1}^{n} H\left(x-Y_{t}^{i, n}\right)$ when $\nu_{n}$ tends to 0 as $n$ tends to infinity. Indeed, denoting by $[y]$ the integer part of any real $y$, one remarks that the approximations $\left|H * \mu_{t}^{n}(x)-[n c] / n\right|$ and $\operatorname{sign}\left(H * \mu_{t}^{n}(x)-[n c] / n\right)\left[\beta\left(H * \mu_{t}^{n}(x)\right)-\beta([n c] / n)\right]$ of the functions which appear in (6) have spatial distribution derivatives respectively equal to $\eta_{t}^{n}=$ $\frac{1}{n} \sum_{i=1}^{n} \operatorname{sign}(i-[c n]) \delta_{Y_{t}^{i, n}}$ and close to $\xi_{t}^{n}=\frac{1}{n} \sum_{i=1}^{n} \operatorname{sign}(i-[c n]) \beta^{\prime}(i / n) \delta_{Y_{t}^{i, n}}$. For $g$ a non-negative test function on $R_{+} \times R$ and $\psi(t, x)=\int_{-\infty}^{x} g(t, y) d y$, computing $\psi\left(t, Y_{t}^{i, n}\right)$ by Itô's formula and summing over $i$ the result multiplied by $\operatorname{sign}(i-[c n]) / n$, one obtains that

$<\eta_{t}^{n}, \psi(t,)>.-<\eta_{0}^{n}, \psi(0,)>.-\int_{0}^{t}<\eta_{s}^{n}, \partial_{s} \psi(s,)>.+<\xi_{s}^{n}, \partial_{x} \psi(s,)>d$.

is equal to the sum of the contribution of the reflection local time term which is non-negative and of the contribution of the Brownian terms which vanishes as $n \rightarrow+\infty$. By making spatial integration by parts, one concludes that the entropic inequality tested against the function $g$ approximately holds. This intuitive reasoning can be made rigourous [18] to prove the following result :

Theorem 2. Assume that $\left(\nu_{n}\right)_{n}$ is a sequence of positive numbers converging to 0 as $n$ tends to infinity. Then the empirical cumulative distribution functions $H * \mu_{t}^{n}(x)$ of the particle systems converge to the unique entropic solution $u(t, x)$ of the inviscid scalar conservation law in the sense given by (4). In addition, the empirical measures $\bar{\mu}^{n}$ of the reordered systems converge in probability to the unique $\bar{P} \in \mathcal{P}(C([0,+\infty), R))$ such that $H * \bar{P}_{t}(x)=u(t, x)$ and that the finite dimensional marginals $\bar{P}_{t_{1}, \ldots, t_{k}}$ are equal to the image of the Lebesgue measure on the interval $[0,1]$ by $y \rightarrow$ $\left(\left(H * \bar{P}_{t_{1}}\right)^{-1}(y), \ldots,\left(H * \bar{P}_{t_{k}}\right)^{-1}(y)\right)$.

Remark 2. Even if this is not rigorous because of the possible discontinuities of the function $u$, the second condition which characterizes $\bar{P}$ in the previous theorem intuitively means that $\bar{P}$ gives full weight to the characteristic curves $X$ along which $t \rightarrow u\left(t, X_{t}\right)$ is constant .

Remark 3. Paper [20] investigates the limit behaviour of the particles

$$
X_{t}^{i, n}=X_{0}^{i}+\nu_{n}^{1 / \alpha} S_{t}^{i}+\int_{0}^{t} \beta^{\prime}\left(H * \mu_{s}^{n}\left(X_{s}^{i, n}\right)\right) d s, 1 \leq i \leq n
$$

where $S^{i}, 1 \leq i \leq n$ are independent symmetric stable processes with index $\alpha \in(1,2)$ independent from the initial positions $\left(X_{0}^{1}, \ldots, X_{0}^{n}\right)$ and $\nu_{n}>0$. When the sequence $\nu_{n}$ is constant and equal to $\nu$ (resp. converges to 0 ), the empirical cumulative distribution functions $H * \mu_{t}^{n}(x)$ converge to the unique weak solution $u$ of $\partial_{t} u=-\nu\left(-\partial_{x x}\right)^{\alpha / 2} u-\partial_{x} \beta(u)$ (resp. the unique entropic solution $u$ of the inviscid scalar conservation law). 


\subsection{General Initial Conditions}

The initial condition $u_{0}$ is now supposed to be the cumulative distribution function of a signed measure $m$ on $R$ with total mass $\|m\| \in(0,+\infty)$ and total variation measure $|m|$. Let us first deal with the non-degenerate case $: \forall u \in$ $R, \alpha^{\prime}(u)>0$. As explained in [15], to associate a single martingale problem with (2), one may introduce $h: R \rightarrow\{-\|m\|,\|m\|\}$ a density of $m$ with respect to the probability measure $|m| /\|m\|$. With any $Q \in \mathcal{P}(C([0,+\infty), R))$, one then associates the signed measure $\tilde{Q}$ with density $h\left(X_{0}\right)$ with respect to $Q$. The time-marginals of $\tilde{Q}$ are denoted by $\left(\tilde{Q}_{t}\right)_{t \geq 0}$. Notice that if $Q_{0}=|m| /\|m\|$ then $\tilde{Q}_{0}(d x)=h(x)|m|(d x) /\|m\|=m(d x)$.

Definition 2. A probability measure $P \in \mathcal{P}(C([0,+\infty), R))$ solves problem (MP) if

$$
\begin{aligned}
& \text { 1. } P_{0}=|m| /\|m\| \\
& \text { 2. } M_{t}^{\varphi}=\varphi\left(X_{t}\right)-\varphi\left(X_{0}\right)-\int_{0}^{t} \alpha^{\prime}\left(H * \tilde{P}_{s}\left(X_{s}\right)\right) \varphi^{\prime \prime}\left(X_{s}\right)+\beta^{\prime}\left(H * \tilde{P}_{s}\left(X_{s}\right)\right) \varphi^{\prime}\left(X_{s}\right) d s \\
& \text { is a P-martingale for any function } \varphi \in C_{b}^{2}(R) .
\end{aligned}
$$

Since $\tilde{P}=P$ when $m$ is a probability measure, this definition of problem (MP) generalizes the previous one.

If $P$ solves (MP), then the function $t \rightarrow E^{P}\left(h\left(X_{0}\right) M_{t}^{\varphi}\right)$ is constant. This implies that $t \rightarrow \tilde{P}_{t}$ is a weak solution of (2). With [21], one deduces that $H * \tilde{P}_{t}(x)$ is a weak solution of (1). Like for monotonic initial conditions $u_{0}$, uniqueness for problem (MP) follows in case $\alpha(u)=\nu u$ with $\nu>0$ [15] and in case $\beta \equiv 0$ (see [9] and Lemma 1 in the appendix).

The particle dynamics generalizing (3) is

$$
X_{t}^{i, n}=X_{0}^{i}+\int_{0}^{t} \sqrt{2 \alpha^{\prime}}\left(H * \tilde{\mu}_{s}^{n}\left(X_{s}^{i, n}\right) d W_{s}^{i}+\int_{0}^{t} \beta^{\prime}\left(H * \tilde{\mu}_{s}^{n}\left(X_{s}^{i, n}\right)\right) d s, i \leq n\right.
$$

where the contribution of the $i$-th particle to the signed empirical measure $\tilde{\mu}^{n}=\frac{1}{n} \sum_{j=1}^{n} h\left(X_{0}^{j}\right) \delta_{X^{j, n}}$ has the weight $h\left(X_{0}^{i}\right)$. Because of the possible irregularity of the function $h$, the choice of initial variables $X_{0}^{i}, 1 \leq i \leq n$ i.i.d. according to $|m| /\|m\|$ is crucial for the validity of the convergence results below. The stochastic differential equation (7) still admits a unique weak solution and the laws $\pi^{n}$ of the empirical measures $\mu^{n}=\frac{1}{n} \sum_{i=1}^{n} \delta_{X^{i, n}}$ form a tight sequence. By an easy adaptation of Proposition 2.4 [18], the limit $\pi^{\infty}$ of any convergent subsequence of $\left(\pi^{n}\right)_{n}$ gives full weight to the probability measures $Q \in \mathcal{P}(C([0,+\infty), R))$ such that $H * \tilde{Q}_{t}(x)$ is a weak solution of (1). When this equation admits a unique weak solution $u(t, x)$, one deduces :

$$
\forall T>0, \lim _{n \rightarrow+\infty} E \sup _{t \in[0, T]} \int_{R} \frac{\left|u(t, x)-H * \tilde{\mu}_{t}^{n}(x)\right|}{1+x^{2}} d x=0 .
$$

Since (5) still holds, one obtains that $\pi^{\infty}$ a.s., $d t$ a.e., $Q_{t}$ does not weight points and concludes that $\pi^{\infty}$ gives full weight to solutions of problem (MP). 
The next theorem puts together the results obtained above and a fluctuation result adapted from [29] in [15] which ensures that the rate of convergence of $H * \tilde{\mu}_{t}^{n}(x)$ to $u(t, x)$ is $1 / \sqrt{n}$ in the case of the viscous scalar conservation law.

Theorem 3. Assume that $\alpha(u)=\nu u$ with $\nu>0$ (resp. that $\alpha^{\prime}$ is a positive function and that $\beta \equiv 0$ ). Then (1) admits a unique solution u (resp. a unique solution $u$ such that $\left\|u(t, .)-u_{0}\right\|_{L^{1}(R)}$ is locally integrable with respect to $t \in R_{+}$and tends to 0 as $t$ tends to 0$)$. As $n$ tend to infinity, the weighted empirical cumulative distribution functions $H * \tilde{\mu}_{t}^{n}(x)$ of the particle systems converge to $u(t, x)$ in the sense given by (8). The empirical measures of the particles converge in probability to the unique solution $P$ of problem (MP). If $\alpha(u)=\nu u$ with $\nu>0$ and the function $\beta$ is $C^{3}$, then the finite-dimensional marginals of the fluctuation field $\left\{\frac{1}{\sqrt{n}} \sum_{i=1}^{n}\left(\varphi\left(X^{i, n}\right)-<P, \varphi()>.\right), \varphi \in\right.$ $\left.L^{2}(P)\right\}$ converge weakly to those of a centered Gaussian field.

Remark 4. In practice, the particles positions $X_{t}^{i, n}$ cannot be computed exactly. The stochastic differential equation giving their evolution has to be discretized with respect to time. Let us denote by $U_{n}(k \Delta t, x)$ the approximate solution of (1) obtained with the standard Euler scheme with timestep $\Delta t$. The convergence estimate $\sup _{k \leq T / \Delta t} E\left\|U_{n}(k \Delta t, x)-u(t, .)\right\|_{L^{1}(R)} \leq$ $C\left(\sqrt{\Delta t}+\frac{1}{\sqrt{n}}\right)$ obtained by Bossy and Talay [7] [8] for the viscous Burgers equation $\left(\alpha(u)=\nu u\right.$ with $\nu>0$ and $\left.\beta(u)=u^{2}\right)$ was extended to general viscous scalar conservation laws and improved into

$$
\sup _{\substack{k \leq T / \Delta t \\ x \in R}} E\left[\left|U_{n}(k \Delta t, x)-u(t, x)\right|+\left\|U_{n}(k \Delta t, x)-u(t, .)\right\|_{L^{1}(R)}\right] \leq C\left(\Delta t+\frac{1}{\sqrt{n}}\right)
$$

by Bossy [4].

Because of the possibility for the paths of particles with opposite weights to intersect, the reordered system no longer evolves according to a diffusion dynamics. In addition, when trying to approximate the entropic inequalities in the vanishing viscosity limit, the contribution of the reflection local time corresponding to such crossings has the wrong sign. To overcome these difficulties, it is possible to modify the particles dynamics by killing the couples of particles with opposite weights that merge [18]. From the point of view of approximation of the equation (1), this idea turns out to be natural. Indeed, if $I_{t} \subset\{1, \ldots, n\}$ denotes the set of indices of particles still alive at time $t$, the approximate solution $U_{n}(t, x)=\frac{1}{n} \sum_{i \in I_{t}} h\left(X_{0}^{i}\right) H\left(x-X_{t}^{i, n}\right)$ is such that $\max _{x \in R} U_{n}(t, x)$ (resp. $\min _{x \in R} U_{n}(t, x)$ ) is non-increasing (resp. non-decreasing) with $t$, property which is a discrete level translation of the maximum principle satisfied by the solutions of (1). In addition, the total variation of $x \rightarrow U_{n}(t, x)$ is non-increasing with $t$. In [18], existence for the particle system with killing

$$
d X_{t}^{i, n}=1_{I_{t}}(i)\left(\sqrt{2 \nu_{n}} d W_{t}^{i}+\beta^{\prime}\left(U_{n}\left(s, X_{s}^{i, n}\right)\right) d t\right), i \leq n
$$


associated with the scalar conservation law is checked and the following convergence result is proved for the approximate solution $U_{n}$ :

Theorem 4. Assume that the sequence $\left(\nu_{n}\right)_{n}$ converges to $\nu \geq 0$. Then

$$
\forall T>0, \lim _{n \rightarrow+\infty} E \sup _{t \in[0, T]} \int_{R} \frac{\left|u(t, x)-U_{n}(t, x)\right|}{1+x^{2}} d x=0,
$$

where $u$ denotes the solution of the viscous scalar conservation law if $\nu>0$ and the entropic solution of the inviscid conservation law otherwise.

\section{Multidimensional Equations}

We are first going to deal with a viscous scalar conservation law in arbitrary space dimension. Then we will consider the incompressible Navier-Stokes equation in space dimension 2.

\subsection{Viscous Scalar Conservation Law}

Let us consider the following viscous scalar conservation law in space dimension $d$.

$$
\begin{gathered}
\partial_{t} u(t, x)=\nu \Delta u(t, x)-\nabla \cdot \beta(u(t, x)), \quad(t, x) \in R_{+} \times R^{d} \\
u(0, x)=u_{0}(x), \quad x \in R^{d}
\end{gathered}
$$

where $\nu>0, \beta: R \rightarrow R^{d}$ is a $C^{2}$ function bounded together with its first and second order derivatives.

By spatial derivation of (9), one obtains formally that for $1 \leq i \leq d$, $v_{i}=\partial_{x_{i}} u$ solves

$$
\partial_{t} v_{i}=\nu \Delta v_{i}-\nabla \cdot\left(\beta^{\prime}(u) v_{i}\right) \text { and } v_{i}(0, x)=\partial_{x_{i}} u_{0}(x) .
$$

To obtain a closed system for $\left(v_{1}, \ldots, v_{d}\right)$, one has to express $u$ in terms of its spatial gradient. In space dimension $d \geq 2$, this question is less obvious than in the previous one-dimensional setting. Anderson [1] first proposed to use the fundamental solution of the Laplacian $\gamma(x)$ for this purpose in a particle method context (see also [13] [28]). One has $\gamma(x)=g(|x|)$ where $g(r)=\log (r) / S_{2}$ if $d=2$ and $g(r)=-1 / S_{d} r^{d-2}$ if $d \geq 3$, with $S_{d}$ denoting the unit sphere area in $R^{d}$. When $f: R^{d} \rightarrow R$ is a $C^{\infty}$ function equal to a constant $c$ outside of a compact set then $f=c+\gamma * \Delta f$. By integration be parts, one deduces that $f=c+\sum_{j=1}^{d} \partial_{x_{j}} \gamma * \partial_{x_{j}} f=c+\nabla \gamma * \nabla f$. This formula is in fact a generalization of the one used in dimension one, since then the derivative of the fundamental solution of the Laplacian $|x| / 2$ is equal to $H-1 / 2$.

We assume from now on that $u_{0}$ is bounded with first order distribution derivatives belonging to $L^{1} \cap L^{\infty}\left(R^{d}\right)$. Then the representation formula $u_{0}=$ 
$c+\nabla \gamma * \nabla u_{0}$ still holds for some real constant $c$. In addition, (9) has a unique classical solution $u(t, x)$ and this solution is such that for any non-negative $t$, $u(t,)=.c+\nabla \gamma * \nabla u(t,).[17]$. Hence the spatial derivatives $\left(\partial_{x_{1}} u, \ldots, \partial_{x_{d}} u\right)$ of $u$ solve weakly the following system :

$$
\partial_{t} v_{i}=\nu \Delta v_{i}-\nabla \cdot\left[\beta^{\prime}\left(c+\sum_{j=1}^{d} \partial_{x_{j}} \gamma * v_{j}\right) v_{i}\right], v_{i}(0, .)=\partial_{x_{i}} u_{0}
$$

As in space dimension one, by spatial derivation we have transformed the equation (9) with local nonlinearity into the previous system with nonlocal nonlinearity. In (9), in the function $\beta$, the unknown $u$ is convoluted with the Dirac mass at the origin, whereas in (10), in the function $\beta^{\prime}$, the unknown $\left(v_{1}, \ldots, v_{d}\right)$ is convoluted with $\nabla \gamma$ which can be seen as the Dirac mass integrated once.

As the solution of (9) can be deduced from the solution of a similar equation where all the spatial coordinates $i \leq d$ such that $\left\|\partial_{x_{i}} u_{0}\right\|_{L^{1}\left(R^{d}\right)}=0$ have been removed, we suppose without restriction that for $i \leq d,\left\|\partial_{x_{i}} u_{0}\right\|_{L^{1}(R)}>0$.

We are now going to give a probabilistic interpretation to the system (10). Since the functions $\partial_{x_{i}} u_{0}$ are not in general probability densities, we associate with any $\left(P^{1}, \ldots, P^{d}\right) \in \mathcal{P}\left(C\left([0,+\infty), R^{d}\right)\right)^{d}$, the measures $\tilde{P}^{i}$ with density $h_{i}\left(X_{0}\right)$ with respect to $P^{i}$ where $h_{i}(x)=\left\|\partial_{x_{i}} u_{0}\right\|_{L^{1}\left(R^{d}\right)} \operatorname{sign}\left(\partial_{x_{i}} u_{0}(x)\right)$ and $\left(X_{t}\right)_{t \geq 0}$ denotes the canonical process on $C\left([0,+\infty), R^{d}\right)$. Let us denote respectively by $\left(P_{t}^{i}\right)_{t \geq 0}$ and $\left(\tilde{P}_{t}^{i}\right)_{t \geq 0}$ the time marginals of the measures $P^{i}$ and $\tilde{P}^{i}$.

Definition 3. $\left(P^{1}, \ldots, P^{d}\right)$ solves problem (MP) if for $1 \leq i \leq d$,

1. $P_{0}^{i}=\frac{\left|\partial_{x_{i}} u_{0}(x)\right|}{\left\|\partial_{x_{i}} u_{0}\right\|_{L^{1}\left(R^{d}\right)}} d x$.

2. For any $t \geq 0, P_{t}^{i}$ has a bounded density w.r.t. the Lebesgue measure on $R^{d}$.

3. $M_{t}^{\varphi}=\varphi\left(X_{t}\right)-\varphi\left(X_{0}\right)-\int_{0}^{t} \nu \Delta \varphi\left(X_{s}\right)+\beta^{\prime}\left(c+\nabla \gamma * \tilde{P}_{s}\left(X_{s}\right)\right) . \nabla \varphi\left(X_{s}\right) d s$ is a $P^{i}$ martingale for any function $\varphi \in C_{b}^{2}\left(R^{d}\right)$ (here $\left.\nabla \gamma * \tilde{P}_{s}=\sum_{j=1}^{d} \partial_{x_{j}} \gamma * \tilde{P}_{s}^{j}\right)$.

If $\left(P^{1}, \ldots, P^{d}\right)$ solves this problem then $t \rightarrow\left(\tilde{P}_{t}^{1}, \ldots, \tilde{P}_{t}^{d}\right)$ is a weak solution of $(10)$.

Because of the explosion of the kernel $\nabla \gamma$ at the origin, it is necessary to introduce a cutoff to construct systems of particles associated with (MP). For $\varepsilon>0$ let $\gamma^{\varepsilon}(x)=g^{\varepsilon}(|x|)$ where

$$
g^{\varepsilon}(r)=g(r) 1_{\{r \geq \varepsilon\}}+\left(\frac{g^{\prime}(\varepsilon) r^{2}}{2 \varepsilon}+g(\varepsilon)-\frac{g^{\prime}(\varepsilon) \varepsilon}{2}\right) 1_{\{0 \leq r<\varepsilon\}} .
$$

Let $\varepsilon_{n}>0$ denote the cutoff parameter ruling the interaction for the system with $n$ particles. Each particle $\left(X_{1}^{i, n}(t), \ldots, X_{d}^{i, n}(t)\right)$ has $d$ coordinates $X_{j}^{i, n}(t)$, $j \leq d$ which evolve in $R^{d}$ according to 


$$
\begin{aligned}
X_{j}^{i, n}(t) & =X_{j}^{i}(0)+\sqrt{2 \nu} W_{t}^{i} \\
& +\int_{0}^{t} \beta^{\prime}\left(c+\sum_{l=1}^{d} \frac{1}{n-1} \sum_{k \neq i} \partial_{x_{l}} \gamma^{\epsilon_{n}}\left(X_{j}^{i, n}(s)-X_{l}^{k, n}(s)\right) h_{l}\left(X_{l}^{k}(0)\right)\right) d s,
\end{aligned}
$$

where $W^{1}, \ldots, W^{n}$ are independent $d$-dimensional Brownian motions which are independent from the $R^{d \times d}$-valued initial variables $\left(X_{1}^{i}(0), \ldots, X_{d}^{i}(0)\right)$, $1 \leq i \leq n$. These initial varibles are supposed to be i.i.d. with $X_{j}^{1}(0)$ distributed according to $\left|\partial_{x_{j}} u_{0}(x)\right| d x /\left\|\partial_{x_{j}} u_{0}\right\|_{L^{1}\left(R^{d}\right)}$ for $1 \leq j \leq d$. The next theorem summarizes the results proved in [17] :

Theorem 5. Problem (MP) has a unique solution $\left(P^{1}, \ldots, P^{d}\right)$. In addition, the unique classical solution $u$ of $(9)$ is such that $\forall(t, x) \in[0,+\infty) \times$ $R^{d}, u(t, x)=c+\nabla \gamma * \tilde{P}_{t}(x)$. If the sequence $\left(\varepsilon_{n}\right)_{n}$ converges to 0 as $n$ tends to $\infty$, then $\left(\frac{1}{n} \sum_{i=1}^{n} \delta_{X_{1}^{i, n}}, \ldots, \frac{1}{n} \sum_{i=1}^{n} \delta_{X_{d}^{i, n}}\right)$ converges in probability to $\left(P^{1}, \ldots, P^{d}\right)$.

As a consequence of this convergence result,

$$
\sup _{(t, x) \in[0, T] \times R^{d}} E\left|u(t, x)-c-\frac{1}{n} \sum_{i=1}^{n} \sum_{j=1}^{d} \partial_{x_{j}} \gamma^{\epsilon_{n}}\left(x-X_{j}^{i, n}(t)\right) h_{j}\left(X_{j}^{i}(0)\right)\right|
$$

converges to 0 as $n$ tends to $\infty$ which ensures that the approximate solution of (9) converges to the exact solution.

\subsection{Incompressible Navier-Stokes Equation in $R^{2}$}

In absence of external forces, the velocity field $u=\left(u^{1}, u^{2}\right)$ and the pressure field $p$ of an incompressible Newtonian fluid in $R^{2}$ solve the following NavierStokes equation

$$
\begin{gathered}
\partial_{t} u=\nu \Delta u-(u \cdot \nabla) u-\nabla p, t \geq 0, x=\left(x_{1}, x_{2}\right) \in R^{2} \\
\nabla \cdot u=0, t \geq 0, x \in R^{2} \\
u(t, x) \rightarrow 0 \text { as }|x| \rightarrow+\infty \text { and } u(0, x)=u_{0}(x), x \in R^{2}
\end{gathered}
$$

It is well known that this equation can be reformulated in terms of the vorticity field : $w=\operatorname{curl} u \stackrel{\text { def }}{=} \partial_{x_{1}} u^{2}-\partial_{x_{2}} u^{1}$. By derivation of (11), one obtains

$$
\partial_{t} w=\nu \Delta w-\nabla \cdot(u w) .
$$

To obtain a closed equation for $w$, one has to express the velocity in terms of the vorticity. Since $\nabla . u=0$, there exists a function $\psi$ such that $u=\nabla^{\perp} \psi=$ $\left(\partial_{x_{2}} \psi,-\partial_{x_{1}} \psi\right)$. On deduces that $w=\partial_{x_{1}}\left(-\partial_{x_{1}} \psi\right)-\partial_{x_{2}}\left(\partial_{x_{2}} \psi\right)=-\Delta \psi$. Hence $\psi=-\gamma * w$ where $\gamma$ denotes the fundamental solution of the Laplacian on $R^{2}$ 
and $u=-\nabla^{\perp} \gamma * w$ is the spatial convolution of the kernel of Biot and Savart $-\nabla^{\perp} \gamma$ with the vorticity field $w$. Finally, the vorticity field solves

$$
\partial_{t} w=\nu \Delta w+\nabla \cdot\left(\left(\nabla^{\perp} \gamma * w\right) w\right) \text { and } w(0, .)=\operatorname{curl} u_{0}(.) .
$$

From now on, we follow Méléard [25] and assume that $m=\operatorname{curl} u_{0}$ is a bounded signed measure on $R^{2}$ with total mass $\|m\|>0$ (see also [23] and [24] for similar results under more restrictive assumptions). For $h: R^{2} \rightarrow\{-\|m\|,\|m\|\}$ a density of $m$ with respect to the probability measure $|m| /\|m\|$, one associates with any $Q \in \mathcal{P}\left(C\left([0,+\infty), R^{2}\right)\right)$ the signed measure $\tilde{Q}$ with density $h\left(X_{0}\right)$ with respect to $Q$ (here $\left(X_{t}\right)_{t \geq 0}$ denotes the canonical process on $\left.\left.C\left([0,+\infty), R^{2}\right)\right)\right)$. The time-marginals of $\tilde{\tilde{Q}}$ are denoted by $\left(\tilde{Q}_{t}\right)_{t \geq 0}$. Interpreting (12) as a Fokker-Planck equation, one associates the following nonlinear martingale problem with this equation

Definition 4. A probability measure $P \in \mathcal{P}\left(C\left([0,+\infty), R^{2}\right)\right)$ solves problem (MP) if

$$
\text { 1. } P_{0}=|m| /\|m\|
$$

2. $M_{t}^{\varphi}=\varphi\left(X_{t}\right)-\varphi\left(X_{0}\right)-\int_{0}^{t} \nu \Delta \varphi\left(X_{s}\right)-\nabla^{\perp} \gamma * \tilde{P}_{s}\left(X_{s}\right) . \nabla \varphi\left(X_{s}\right) d s$ is a $P$ martingale for any function $\varphi \in C_{b}^{2}\left(R^{2}\right)$.

Because of the singularity of $\nabla^{\perp} \gamma$ at the origin, it is necessary to cutoff this kernel in order to construct associated interacting particle systems. To keep important features of $\nabla^{\perp} \gamma$ such as the divergence-free property, one defines $k_{\varepsilon}=-\nabla^{\perp} \gamma * \phi_{\varepsilon}$ where $\phi_{\varepsilon}(x)=\phi(x / \varepsilon) / \varepsilon^{2}$ with $\phi$ a smooth function with radial symmetry and integral equal to 1 . This way, $k_{\varepsilon}$ is bounded by $M_{\varepsilon}$ and Lipschitz continuous with constant $L_{\varepsilon}$. One may choose $M_{\varepsilon}=O\left(1 / \varepsilon^{2}\right)$ and $L_{\varepsilon}=O\left(1 / \varepsilon^{3}\right)$ as $\varepsilon$ tends to 0.

For $W^{1}, \ldots, W^{n}$ independent 2-dimensional Brownian motions independent from the initial variables $X_{0}^{1}, \ldots, X_{0}^{n}$ i.i.d. according to $|m| /\|m\|$, the system with $n$ particles is defined by

$$
X_{t}^{i, n}=X_{0}^{i}+\sqrt{2 \nu} W_{t}^{i}+\int_{0}^{t} k_{\varepsilon_{n}} * \tilde{\mu}_{s}^{n}\left(X_{s}^{i, n}\right) d s, 1 \leq i \leq n
$$

where $\tilde{\mu}_{s}^{n}=\frac{1}{n} \sum_{j=1}^{n} h\left(X_{0}^{j}\right) \delta_{X_{s}^{j, n}}$ and $\varepsilon_{n}>0$. According to [25],

Theorem 6. Existence holds for problem (MP). The solution $P$ is unique when either $m$ does not weight points or $\|m\|$ is small enough.

When uniqueness holds, for $T>0$, if

$$
\lim _{n \rightarrow+\infty} \varepsilon_{n}=0 \text { and } \lim _{n \rightarrow+\infty} M_{\varepsilon_{n}} e^{\|m\| L_{\varepsilon_{n}} T} / \sqrt{n} L_{\varepsilon_{n}}=0,
$$

then the empirical measures $\mu^{n}=\frac{1}{n} \sum_{i=1}^{n} \delta_{X^{i, n}}$ considered as random variables with values in $\mathcal{P}\left(C\left([0, T], R^{2}\right)\right)$ converge in probability to $P \circ\left(\left(X_{t}\right)_{t \in[0, T]}\right)^{-1}$. 
When $u_{0}$ is such that $\sup _{y>0} y\left(\operatorname{mes}\left(\left\{x:\left|u_{0}(x)\right|>y\right\}\right)\right)^{1 / 2}<+\infty, \nabla \cdot u_{0}=0$ and $m=$ curl $u_{0}$ either does not weight points or has a small enough total mass, then the Navier-Stokes equation (11) admits a unique solution $(u, p)$. And it is possible to approximate the velocity field thanks to the particle system since under $(13)$, for $(t, x) \in[0, T] \times R^{2}, \frac{1}{n} \sum_{i=1}^{n} h\left(X_{0}^{i}\right) k_{\varepsilon_{n}}\left(x-X_{t}^{i, n}\right)$ converges in probability to $u(t, x)$.

\section{Bounded Spatial Domains}

We are first going to deal with a viscous scalar conservation law posed on the spatial interval $[0,1]$ before considering the incompressible Navier-Stokes equation in a bounded domain of $R^{2}$.

\subsection{Viscous Scalar Conservation Law in the Spatial Interval $[0,1]$}

We are now interested in the following viscous scalar conservation law posed in the spatial interval $[0,1]$ with non-homogeneous Dirichlet boundary conditions

$$
\begin{gathered}
\partial_{t} u(t, x)=\nu \partial_{x x}^{2} u(t, x)-\partial_{x} \beta(u(t, x)),(t, x) \in R_{+} \times(0,1) \\
\forall x \in[0,1], u(0, x)=u_{0}(x) \text { and } \forall t \geq 0, u(t, 0)=0 \text { and } u(t, 1)=1
\end{gathered}
$$

where $u_{0}$ is the cumulative distribution function of a probability measure $m$ on the interval $[0,1]$. The probabilistic interpretation of this equation involves a diffusion process with normal reflection at the boundary of the interval $[0,1]$. That is why we introduce $\left(X_{t}, K_{t}\right)$ the canonical process on $\mathcal{C}=C([0,+\infty),[0,1]) \times C([0,+\infty), R)$. For $P$ a probability measure on $\mathcal{C}$, we set $\hat{P}_{t}=P \circ X_{t}^{-1}$.

Definition 5. A probability measure $P \in \mathcal{P}(\mathcal{C})$ solves problem (MP) if

$$
\begin{aligned}
& \text { 1. } P \circ\left(X_{0}, K_{0}\right)^{-1}=m \otimes \delta_{0} \\
& \text { 2. } \varphi\left(X_{t}-K_{t}\right)-\varphi\left(X_{0}-K_{0}\right)-\int_{0}^{t} \nu \varphi^{\prime \prime}\left(X_{s}-K_{s}\right)+\beta^{\prime}\left(H * \hat{P}_{s}\left(X_{s}\right)\right) \varphi^{\prime}\left(X_{s}-K_{s}\right) d s \\
& \text { is a } P \text { martingale for any } \varphi \in C_{b}^{2}(R) \text {. } \\
& \text { 3. } P \text { a.s. } \forall t \geq 0,|K|_{t}=\int_{0}^{t} 1_{\{0,1\}}\left(X_{s}\right) d|K|_{s}<+\infty \text { and } K_{t}=\int_{0}^{t} 1_{\left\{X_{s}=0\right\}}- \\
& 1_{\left\{X_{s}=1\right\}} d|K|_{s} \text {. }
\end{aligned}
$$

The process $K_{t}$ with finite variation which increases when $X_{t}$ is equal to 0 and decreases when $X_{t}$ is equal to 1 accounts for reflection and prevents $X_{t}$ from leaving the interval $[0,1]$.

The associated particles are also reflected at the boundary of interval $[0,1]$ :

$$
\begin{gathered}
X_{t}^{i, n}=X_{0}^{i}+\sqrt{2 \nu} W_{t}^{i}+\int_{0}^{t} \beta^{\prime}\left(H * \hat{\mu}_{s}^{n}\left(X_{s}^{i, n}\right)\right) d s+K_{t}^{i, n}, 1 \leq i \leq n \\
\left|K^{i, n}\right|_{t}=\int_{0}^{t} 1_{\{0,1\}}\left(X_{s}^{i, n}\right) d|K|_{s}^{i, n} \text { and } K_{t}^{i, n}=\int_{0}^{t} 1_{\left\{X_{s}^{i, n}=0\right\}}-1_{\left\{X_{s}^{i, n}=1\right\}} d\left|K^{i, n}\right|_{s} \\
\mu^{n}=\frac{1}{n} \sum_{j=1}^{n} \delta_{\left(X^{j, n}, K^{j, n}\right)} \text { and } \hat{\mu}_{s}^{n}=\frac{1}{n} \sum_{j=1}^{n} \delta_{X_{s}^{j, n}} .
\end{gathered}
$$

The next theorem states some of the results proved in [6] 
Theorem 7. Problem (MP) has a unique solution P. In addition, $u(t, x)=$ $H * \hat{P}_{t}(x)$ is a weak solution of (14).

As $n$ tends to infinity, the empirical measures $\mu^{n}$ converge in probability to $P$ and

$$
\forall(t, x) \in R_{+} \times[0,1], \lim _{n \rightarrow+\infty} E\left|u(t, x)-H * \hat{\mu}_{t}^{n}(x)\right|=0 .
$$

To obtain a practical algorithm, the stochastic differential equation giving the evolution of the positions $X_{t}^{i, n}$ has to be discretized with respect to time. The main part of [6] is dedicated to the numerical analysis of the algorithm obtained by using for this purpose the version of the Euler scheme with step $\Delta t$ proposed by Lépingle [22]. To precise this scheme, one needs two constant $\alpha_{0}<$ $\alpha_{1}$ in $(0,1)$. As in a standard Euler scheme, the value of the drift coefficient of each particle is frozen on each time-step to a value depending on the positions of all particles at the beginning of the time-step. If the position of the $i$ th particle at the beginning of a time-step is in $\left[\alpha_{0}, \alpha_{1}\right]$, then one computes the value of the process with frozen drift coefficient and without reflection at the end of the time-step. If the position is in $\left[0, \alpha_{0}\right)$ (resp. $\left.\left(\alpha_{1}, 1\right]\right)$ then one computes the value of the process with frozen drift coefficient normally reflected at 0 (resp. 1 ). In each case, the result is projected on $[0,1]$ to obtain the position of the $i$-th particle at the beginning of the next time-step.

Let us denote by $\bar{X}_{k \Delta t}^{i, n}, 1 \leq i \leq n, 0 \leq k \leq T / \Delta t$ the discretized positions of the particles obtained thanks to this scheme. The following convergence rate is proved in [6] :

$$
\sup _{\substack{x \in[0,1] \\ k \leq T / \Delta t}} E\left|\frac{1}{n} \sum_{i=1}^{n} H\left(x-\bar{X}_{k \Delta t}^{i, n}\right)-u(k \Delta t, x)\right| \leq C\left(\Delta t+\frac{1}{\sqrt{n}}\right) .
$$

This rate is the same as the one obtained before by Bossy [4] when the spatial domain is the whole real line i.e. in the absence of reflection of particles .

On the present example, because of the appropriate choice of the boundary conditions at positions 0 and 1, everything works as if the spatial domain was the whole real line. Let us now turn to an example in which the boundary conditions come from the physics.

\subsection{Incompressible Navier-Stokes Equation in a Bounded Domain}

We are interested in the incompressible Navier-Stokes equation in a bounded domain $\Theta$ of $R^{2}$ with no-slip boundary conditions on $\partial \Theta$ :

$$
\begin{gathered}
\partial_{t} u=\nu \Delta u-(u \cdot \nabla) u-\nabla p, t \geq 0, x \in \Theta \\
\nabla \cdot u=0, t \geq 0, x \in \Theta \\
u(0, x)=u_{0}(x), x \in \Theta \text { and } u(t, x)=0, t \geq 0, x \in \partial \Theta .
\end{gathered}
$$

As when the spatial domain is the whole plane, the vorticity field $w=\operatorname{curl} u$ solves 


$$
\partial_{t} w=\nu \Delta w-\nabla \cdot(u w) \text { and } w(0, .)=\operatorname{curl} u_{0}
$$

To obtain a closed equation for $w$, one faces two difficulties. First, as usual, one has to express $u$ in terms of $w$. Second, one has to translate the no-slip boundary condition for the velocity field in terms of a boundary condition for the vorticity field. Of course, both issues are closely related.

Since $\nabla . u=0$, one has $u=\nabla^{\perp} \psi$ and $w=-\Delta \psi$. One may choose $\psi(t, x)=$ $-\int_{\Theta} \gamma(x, y) w(t, y) d y$ where $\gamma$ is the Green function of the Laplacian on $\Theta$ with homogeneous Dirichlet boundary conditions. One deduces

$$
u=-\left[\nabla^{\perp} \gamma w\right] \text { where }-\left[\nabla^{\perp} \gamma w\right](t, x) \text { stands for }-\int_{\Theta} \nabla_{x}^{\perp} \gamma(x, y) w(t, y) d y .
$$

Because of the choice of homogeneous Dirichlet boundary conditions, $\psi$ vanishes on $\partial \Theta$. As a consequence, the tangential derivative of $\psi$ is zero. Since $u=\nabla^{\perp} \psi$, the normal component of the velocity vanishes on the boundary. As pointed out by Chorin [11], to ensure that the tangential component of the velocity is also zero, vorticity has to be created on the boundary. The following Neumann's boundary condition involving a nonlocal right-hand-side proposed by Cottet [12] ensures the correct creation of vorticity

$$
\partial_{n} w=\partial_{n} \operatorname{curl} \gamma\left(-\partial_{2} w, \partial_{1} w\right)-\frac{1}{|\partial \Theta|} \int_{\partial \Theta} \partial_{n} \operatorname{curl} \gamma\left(-\partial_{2} w, \partial_{1} w\right), t \geq 0, x \in \partial \theta .
$$

Here $\partial_{n}$ denotes the normal component of the gradient and

$$
\gamma\left(-\partial_{2} w, \partial_{1} w\right)(t, x)=\left(-\int_{\Theta} \gamma(x, y) \partial_{2} w(t, y) d y, \int_{\Theta} \gamma(x, y) \partial_{1} w(t, y) d y\right) .
$$

This condition of Neumann's type seems well-suited for the probabilistic interpretation since it can be translated into mass creation on the boundary.

In [19], as a first step towards this probabilistic interpretation we deal with the vortex equation

$$
\partial_{t} w=\nu \Delta w+\nabla \cdot\left(\left[\nabla^{\perp} \gamma w\right] w\right) \text { and } w(0, .)=w_{0}(.)
$$

with initial condition $w_{0} \in L^{2}(\Theta)$ supplemented by the Neumann's boundary condition

$$
\partial_{n} w(t, x)=g(t, x), t \geq 0, x \in \partial \Theta
$$

where $g$ is a given function belonging to $L^{2}([0, T] \times \partial \Theta)$ with $T>0$ a finite time horizon. We associate with this equation a nonlinear martingale problem on a space of reflected paths either starting initially from $\Theta$ or starting from $\partial \Theta$ after that. We show uniqueness for the martingale problem and prove the convergence of the empirical measures of well-chosen particle systems to the solution of the martingale problem. Finally, we show that the corresponding velocity field $-\left[\nabla^{\perp} \gamma w\right]$ can be approximated thanks to the particles (see [19] for details). 
Next, we have tried to deal with the nonlocal boundary condition proposed by Cottet. Since, to our knowledge, no energy estimate is available for the vortex equation supplemented by this boundary condition, the theoretical study appears really difficult. And so far, we have not been able to obtain convincing numerical results. Notice that a different probabilistic interpretation based on branching processes was developped by Benachour, Roynette and Vallois [3] for (15). But even if the authors propose some particle approximations, the convergence of the method is not shown and the particle systems are not for use in practice.

\section{Conclusion}

For all the parabolic evolution equations with local nonlinearity treated in the present paper, we have shown that a suitable derivation of the solution with respect to spatial variables enables to obtain a closed equation (or a closed system of equations) of Fokker-Planck type with nonlocal nonlinearity : the unknown function is convoluted with a kernel obtained by some spatial integration of the Dirac mass at the origin. In space dimension one, this kernel is equal to the Heaviside function : it is discontinuous at the origin but since it is bounded, its spatial convolution with any bounded signed measure makes sense. This makes the probabilistic interpretation of the equation obtained by derivation easy and enables to obtain rather general approximation results for the solution of the original equation. In space dimension $d \geq 2$, the kernel is singular at the origin. Even if, from a probabilistic point of view, such a kernel is much easier to take into account than the Dirac mass at the origin, one has to be more cautious than in space dimension one.

We have also explained how the use of signed weights depending on the initial positions both in the nonlinear martingale problem and in the particle dynamics allows to give a probabilistic interpretation and design particle approximations for an enlarged class of initial conditions.

Last, we have seen that when the original equation is posed in a bounded spatial domain, the treatment of its boundary conditions after spatial derivation can be rather delicate.

\section{Appendix}

Lemma 1. Let $P$ solve problem (MP) given in Definition 2. Then,

$\forall t \geq 0,\left\|H * \tilde{P}_{t}(.)-u_{0}\right\|_{L^{1}(R)} \leq\|m\|\left(t \sup _{|u| \leq\|m\|}\left|\beta^{\prime}(u)\right|+\sqrt{2 t \sup _{|u| \leq\|m\|}\left|\alpha^{\prime}(u)\right|}\right)$.

Proof. Let $\left(P^{y}\right)_{y \in R}$ be a regular conditional probability distribution of $P$ given $X_{0}=y$. One has 


$$
\begin{aligned}
& \left\|H * \tilde{P}_{t}(.)-u_{0}\right\|_{L^{1}(R)}=\int_{R}\left|\int_{R}\left(P^{y}\left(X_{t} \leq x\right)-1_{\{y \leq x\}}\right) m(d y)\right| d x \\
\leq & \int_{R} \int_{R} 1_{\{y>x\}} P^{y}\left(X_{t}-y \leq x-y\right)+1_{\{y \leq x\}} P^{y}\left(X_{t}-y>x-y\right) d x|m|(d y) \\
= & \int_{R}<P^{y},\left|X_{t}-y\right|>|m|(d y) .
\end{aligned}
$$

One concludes by remarking that $|m|(d y)$ a.e., under $P^{y}$, the canonical process $\left(X_{t}\right)_{t \geq 0}$ solves weakly

$$
X_{t}=y+\int_{0}^{t} \sqrt{2 \alpha^{\prime}}\left(H * \tilde{P}_{s}\left(X_{s}\right)\right) d W_{s}+\int_{0}^{t} \beta^{\prime}\left(H * \tilde{P}_{s}\left(X_{s}\right)\right) d s
$$

which ensures that

$$
<P^{y},\left|X_{t}-y\right|>\leq\left(t \sup _{|u| \leq\|m\|}\left|\beta^{\prime}(u)\right|+\sqrt{2 t \sup _{|u| \leq\|m\|}\left|\alpha^{\prime}(u)\right|}\right) .
$$

\section{References}

1. C.R. Anderson. A vortex method for flows with slight density variations. J. Comp. Physics, 61:417-444, 1985.

2. R.F. Bass and E. Pardoux. Uniqueness for Diffusions with Piecewise Constant Coefficients. Probab. Theory and Related Fields, 76:557-572, 1987.

3. S. Benachour, B. Roynette, and P. Vallois. Branching process associated with 2d Navier-Stokes equation. Rev. Mat. Iberoamericana, 17(2):331-373, 2001.

4. M. Bossy. Optimal rate of convergence of a stochastic particle method for the solution of a 1d viscous scalar conservation law. Math. Comput., 73(246):777$812,2004$.

5. M. Bossy, L. Fezoui, and S. Piperno. Comparison of a stochastic particle method and a finite volume deterministic method applied to Burgers equation. Monte Carlo Methods Appl., 3(2):113-140, 1997.

6. M. Bossy and B. Jourdain. Rate of convergence of a particle method for the solution of a $1 \mathrm{~d}$ viscous scalar conservation law in a bounded interval. Ann. Probab., 30(4):1797-1832, 2002.

7. M. Bossy and D. Talay. Convergence rate for the approximation of the limit law of weakly interacting particles: application to the Burgers equation. Ann. Appl. Probab., 6(3):818-861, 1996.

8. M. Bossy and D. Talay. A stochastic particle method for the McKean-Vlasov and the Burgers equation. Math. Comp., 66(217):157-192, 1997.

9. H. Brezis and M.G. Crandall. Uniqueness of solutions of the initial-value problem for $u_{t}-\Delta \phi(u)=0$. J. Math. pures et appl., 58:153-163, 1979.

10. P. Calderoni and M. Pulvirenti. Propagation of chaos for Burgers' equation. Ann. Inst. Henri Poincaré Section A, 39(1):85-97, 1983.

11. A.J. Chorin. Numerical study of slightly viscous flows J. Fluid Mech. 57:785793, 1973. 
12. G.H. Cottet. A vorticity creation algorithm, Mathematical aspects of vortex dynamics, SIAM, Philadelphia PA, 1989.

13. A.L. Fogelson. Particle-method solution of two-dimensional convection-diffusion equations. J. Comp. Physics, 100:1-16, 1992.

14. B. Jourdain. Diffusions with a nonlinear irregular drift coefficient and probabilistic interpretation of generalized Burgers' equations. ESAIM, Probab. Stat. (http://www.emath.fr/ps/), 1:339-355, 1997.

15. B. Jourdain. Diffusion processes associated with nonlinear evolution equations for signed measures. Methodol. Comput. Appl. Probab., 2(1):69-91, 2000.

16. B. Jourdain. Probabilistic approximation for a porous medium equation. Stochastic Process. Appl., 89(1):81-99, 2000.

17. B. Jourdain. Probabilistic gradient approximation for a viscous scalar conservation law in space dimension $d \geq 2$. Stochastics Stochastics Rep., 71:243-268, 2001.

18. B. Jourdain. Probabilistic characteristics method for a $1 \mathrm{~d}$ scalar conservation law. Ann. Appl. Probab., 12(1):334-360, 2002.

19. B. Jourdain and S. Méléard. Probabilistic interpretation and particle method for vortex equations with Neumann's boundary condition. Proc. Edinburgh Math. Soc., 47(3), pp 597-624, 2004.

20. B. Jourdain, S. Méléard, and W. Woyczynski. Probabilistic approximation and inviscid limits for 1-d fractional conservation laws. Bernoulli, to appear.

21. N.V. Krylov. Some estimates of the probability density of a stochastic integral. Math. USSR Izvestija, 8(1):233-254, 1974.

22. D. Lépingle. Euler scheme for reflected stochastic differential equations. Math. Comput. Simul., 38(1-3):119-126, 1995.

23. C. Marchioro and M. Pulvirenti. Hydrodynamics in two dimensions and vortex theory. Comm. Math. Phys., 84:483-503, 1982.

24. S. Méléard. A trajectorial proof of the vortex method for the two-dimensional Navier Stokes equations. Ann. Appl. Probab., 10(4):1197-1211, 2000.

25. S. Méléard. Monte-Carlo approximations for 2d Navier-Stokes equation with measure initial data. Probab. Theory Relat. Fields, 121:367-388, 2001.

26. K. Oelschläger. A law of large numbers for moderately interacting diffusion processes. Z. Warsch. Verw. Geb., 69:279-322, 1985.

27. B. Roynette and P. Vallois. Instabilité de certaines equations différentielles stochastiques non linéaires. J. Funct. Anal., 130(2):477-523, 1995.

28. A. Sherman and M. Mascagni. A gradient random walk method for twodimensional reaction-diffusion equations. SIAM J. Sci. Comput., 15(6):12801293, november 1994.

29. T. Shiga and H. Tanaka. Central Limit Theorem for a System of Markovian Particles with Mean Field Interactions. Z. Warsch. Verw. Geb., 69:439-459, 1985.

30. D.W. Stroock and S.R.S. Varadhan. Multidimensional Diffusion Processes. Springer, 1997.

31. A.S. Sznitman. A propagation of chaos result for Burgers' Equation. Probab. Theory Relat. Fields, 71:581-613, 1986.

32. A.S. Sznitman. Topics in propagation of chaos. In Ecole d'été de probabilités de Saint-Flour XIX - 1989, Lect. Notes in Math. 1464. Springer-Verlag, 1991. 